\title{
Electron doping in the honeycomb bilayer superconductors (Zr, $\mathrm{Hf}) \mathrm{NCl}$
}

\author{
R. Weht ${ }^{1,2}$, A. Filippettit ${ }^{1}$ and W. E. Picketr ${ }^{1}$ \\ 1 Department of Physics, University of California - Davis CA, 95616 USA \\ 2 Departamento de Física, CNEA - Avenida General Paz y Constituyentes \\ 1650 - San Martín, Argentina
}

(received 24 March 1999; accepted in final form 8 September 1999)

PACS. 74.70Ad - Metals; alloys and binary compounds (including A15, Laves phases, etc.). PACS. 74.25Jb - Electronic structure.

PACS. 74.25Kc - Phonons.

\begin{abstract}
Based on a virtual crystal treatment, we show that alkali doping introduces electron carriers into a single light mass $\left(m^{*}=0.6\right)$, low density of states band in $\mathrm{Li}_{x} \mathrm{ZrNCl}$ and $\mathrm{Na}_{x} \mathrm{HfNCl}$ (which have superconducting $T_{\mathrm{c}}$ up to $25 \mathrm{~K}$ ) that is a symmetric interlayer combination of $d_{x y}, d_{x^{2}-y^{2}}$ character. Doping leads to simple $K$-centered three-fold symmetrical cylindrical Fermi surfaces that concentrate low-energy scattering into distinct regions of momentum. The lack of any observed magnetic tendencies, together with our calculation of small electron-phonon strength, suggests a pairing mechanism that is distinct from known superconductors.
\end{abstract}

Superconductivity in doped quasi-two-dimensional (2D) compounds has been of intense interest since the discovery of the strong dependence of the critical temperature, $T_{\mathrm{c}}$, on intercalation of the transition metal dichalcogenides [1], and the discovery of high $T_{\mathrm{c}}$ in $2 \mathrm{D}$ cuprates further heightened their study. The manner in which doping of 2D layers alters their electronic behaviour varies greatly depending on the system. Cuprate high-temperature superconductors, for instance, have a magnetic insulator to metal transition that completely modifies their electronic structure and continues to attract a huge amount of study [2]. In the dichalcogenides there is minimal magnetic behaviour, and carrier doping occurs in a rigid-band fashion. The fulleride superconductors $\mathrm{A}_{3} \mathrm{C}_{60}(\mathrm{~A}=$ alkali $)$ provide another example in which rigid-band doping gives a realistic picture [3] of a relatively high critical temperature $\left(T_{\mathrm{c}}\right)$ superconductor.

Superconductivity up to $12 \mathrm{~K}$ in Li-doped $\mathrm{ZrNCl}$ was found by Yamanaka et al. [4] (and extended by Shamoto et al. [5]), suggesting a behaviour related to the dichalcogenides, since $T_{\mathrm{c}}$ was in the same range. However, the increase up to $T_{\mathrm{c}}=25 \mathrm{~K}$ in $\mathrm{Na}_{x} \mathrm{HfNCl}(x \sim$ $0.29)[6,7]$ provides impetus to reevaluate this system, since it became the third highest value of $T_{\mathrm{c}}$ outside of the layered cuprate superconductors.

In this paper we present several results that we think are very important in furthering the theoretical understanding of this system: 1) for values of doping where the compounds 

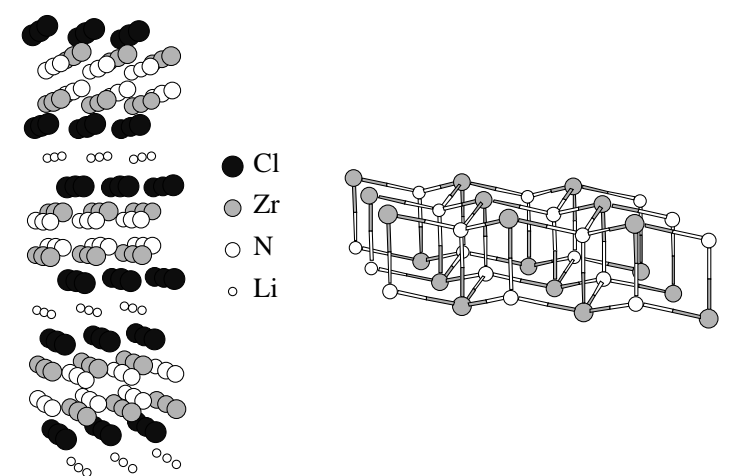

Fig. 1. - Rhombohedral structure of $\mathrm{Li}_{x} \mathrm{ZrNCl}$ determined by Shamoto et al. [5,7]. The right side provides a detail of the $\mathrm{ZrN}$ bilayer.

show superconductivity a single band is occupied, with no interband optical transitions below $\sim 1 \mathrm{eV} ; 2)$ the low mass and high velocity of the carriers indicate that this is not a highly correlated system; 3) evaluations of the London penetration depth and the coherence length indicate type-II superconductivity; 4) the Fermi surface is simple and concentrates scattering processes around certain wave vectors; 5) there are high-energy vibrations of almost pure $\mathrm{N}$ character; 6) estimates give small electron-phonon scattering strength that can not account for $T_{\mathrm{c}} \approx 25 \mathrm{~K}$. These facts together with the high value of $T_{\mathrm{c}}$ and that the insulating parent phase is a rather wide gap, non-magnetic insulator ( $\mathrm{ZrNCl}$ has a $3 \mathrm{eV}$ optical gap [8]) suggest this is a new class of superconductor whose source of pairing is rather mysterious.

There have been a variety of crystal structures reported for $\mathrm{ZrNCl}$. Although all have strongly layered features, the differences are severe enough to strongly affect the electronic structure. We report here on calculations performed using the structures of $\mathrm{Li}_{x} \mathrm{ZrNCl}$ and $\mathrm{Na}_{x} \mathrm{HfNCl}$ reported by Shamoto et al. $[5,7]$ and shown schematically in fig. 1, determined by powder neutron diffraction on superconducting samples [9]. This structure consists of bilayers of $\mathrm{ZrN}$, with $\mathrm{Cl}$ layers lying on each side of the $\mathrm{ZrN}$ sheets. Each neutral $\mathrm{Cl}-\mathrm{ZrN}-\mathrm{ZrN}-\mathrm{Cl}$ structural unit is van der Walls bonded to neighboring units, and the intercalated alkali atoms lie between the $\mathrm{Cl}$ layers. Within one-half of the bilayer, the $\mathrm{Zr}$ and $\mathrm{N}$ ions form two interconnected dimpled honeycomb lattices with the $\mathrm{Zr}$ and $\mathrm{N}$ heights differing by $0.39 \AA$. Across the $\mathrm{ZrN}$ bilayer each $\mathrm{Zr}$ is bonded to a $\mathrm{N}$ in the other layer with a bondlength of $2.13 \AA$. Doping can alter slightly the stacking sequence of the $\mathrm{ZrNCl}$ bilayers, but we find it has minor effect on the electronic structure over most of the reported doping range.

We have studied the electronic and vibrational behaviour using two methods. The linearized augmented plane wave (LAPW) method [10] was used for the virtual crystal studies of doping, and the ultrasoft pseudopotential (USP) method [11] for structural relaxation and calculation of phonon frequencies. Both are full potential methods, which is important for anisotropic, nonclose-packed structures, and give essentially identical results for the undoped materials. The pseudopotentials in the USP methods were tested in other applications, and the $\mathrm{N}$ potential has been used in extensive studies of structural and magnetic properties of $\mathrm{CrN}$ [12] and FeN [13]. The local density approximation (LDA) exchange-correlation potential of Ceperley and Alder was used [14]. Generalized gradient corrections [15] changed eigenvalues only slightly, increasing the gap as is known to be a general trend. Self-consistency on $k$-point meshes up to 1000 points in the Brillouin zone established converged $k$-space sampling.

The electronic structure of $\mathrm{ZrNCl}$ is well characterized by the formal ionic description 


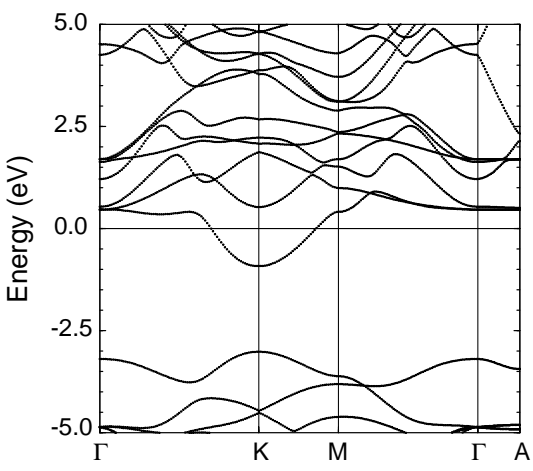

Fig. 2. - Band structure of $\mathrm{Na}_{0.25} \mathrm{HfNCl} . \quad \Gamma=(0,0,0), K=(2 / 3,1 / 3,0), M=(1 / 2,0,0), A=$ $(0,0,1 / 2)$, in units of the hexagonal reciprocal lattice vectors. Notice the lack of dispersion along $\Gamma-A$ of the lower conduction bands.

$\mathrm{Zr}^{+4} \mathrm{~N}^{-3} \mathrm{Cl}^{-1}$. Zr-N covalency is strong, however, so it is better thought of as a band insulator than as a purely ionic insulator. $\mathrm{Cl}$ and $\mathrm{N} 2 p$ states are mixed in the valence band region, with a combined bandwidth of $\sim 5.5 \mathrm{eV}$. The band gap of $1.7 \mathrm{eV}$ is indirect between $\Gamma$ and $K$, and the minimum direct gap of $1.9 \mathrm{eV}$ lies at $K$. The $\mathrm{HfNCl}$ band structure is similar, with indirect (direct) gap of $1.8 \mathrm{eV}(2.1 \mathrm{eV})$. The electronic structure is dominated by the hexagonal bilayer, so we use the more familiar hexagonal symmetry directions and notation rather than the rhombohedral ones.

The intercalating alkali atoms are ionized, with the carriers going into the conduction bands of the undoped compound. Doping by $\mathrm{Na}$ in $\mathrm{Na}_{x} \mathrm{HfNCl}$, for example, is treated within a virtual crystal approximation, where a fractional occupation $\nu$ of a site with monovalent $\mathrm{Na}$ is treated by fully occupying the site with nuclear charge $10+\nu$, hence valence $\nu$. The slight atomic rearrangements upon doping [7] have been neglected for simplicity. Up to a doping level $x=0.35$, occupation of the Hf $d$ bands by the added carriers is rigid-band-like, justifying a posteriori our virtual crystal treatment. Only at a higher doping level $(x=0.5)$ does non-rigid-band behavior become important.

As is clear from fig. 2, the carriers fill a single band with minima at the three inequivalent zone corner $(K)$ points. From the projected density of states in fig. 3, this band is seen to be of mixed Hf $d_{x y}, d_{x^{2}-y^{2}}$ and $\mathrm{N} p_{x}, p_{y}$ character, and lies $1 \mathrm{eV}$ below the remaining $d$ bands (10 in total). Since the two $d_{x y}, d_{x^{2}-y^{2}}$ states are degenerate in the $3 m$ symmetry of the transition metal ion, and there are two such ions per cell, there are four bands of this character. Two of them are the lowest two bands at $K$ and are split by $1 \mathrm{eV}$ into symmetric and antisymmetric bilayer bands; the other two lie $\sim 4 \mathrm{eV}$ above $E_{\mathrm{F}}$ and mix strongly with other bands. These features are similar to those calculated by Felser and Seshadri [16] for undoped $\mathrm{ZrNCl}$ in a related structure.

Doping by Na up to the level we have studied (apparently close to the optimal doping level) puts carriers only into the symmetric (bonding) band. The value of $E_{\mathrm{F}}=1.0 \mathrm{eV}$ and the FS dimensions gives a light band mass $m_{\mathrm{b}} \approx 0.6$; the bands are actually sub-parabolic around the Fermi energy $E_{\mathrm{F}}$. The Fermi level density of states $N\left(E_{\mathrm{F}}\right)=0.74$ states/eV is very low for a good superconductor, for example it is only one-third of the value for $\mathrm{La}_{0.85} \mathrm{Sr}_{0.15} \mathrm{CuO}_{4}$ which also is a doped insulator with a single band crossing $E_{\mathrm{F}}$ and $T_{\mathrm{c}} \sim 25-40 \mathrm{~K}$ [17].

Since the reported doping level $x=0.29$ is only approximate, in the following we quote representative materials parameters for $x=0.25-0.30$. Two properties that can be obtained 

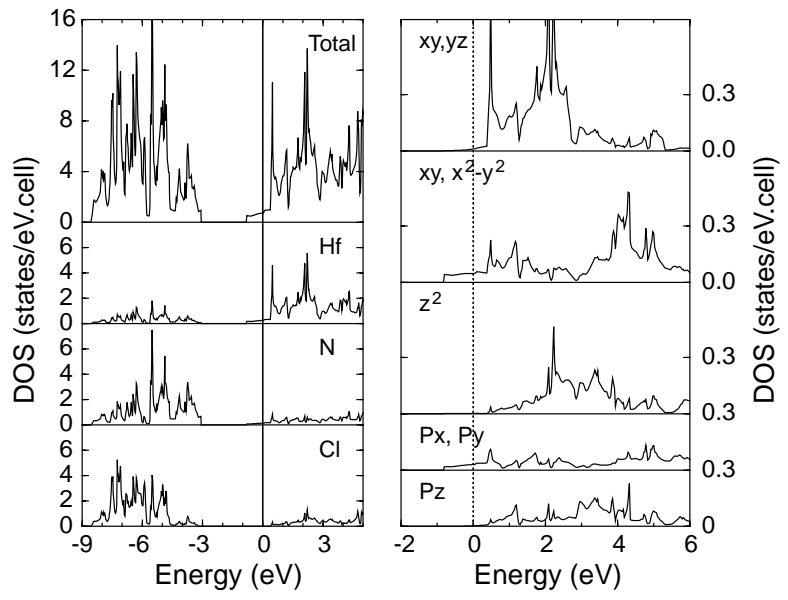

Fig. 3. - Projected densities of states for $\mathrm{Na}_{0.25} \mathrm{HfNCl}$. Left panel: total atomic contributions. Right panel: Hf $d$ and N $p$ DOS separated into their orbital contributions.

immediately are the superconducting coherence length $\xi$ and the field penetration depth $\Lambda$ (both in-plane), given by

$$
\xi=\frac{\hbar v_{\mathrm{F}}}{\pi \Delta}, \quad \Lambda=\frac{c}{\Omega_{\mathrm{p}}} ; \quad \Omega_{\mathrm{p}}^{2}=4 \pi e^{2} N\left(E_{\mathrm{F}}\right) v_{\mathrm{F}, x}^{2} .
$$

$\Omega_{\mathrm{p}}$ is the plasma frequency and can be estimated from our data to be around $1.5 \mathrm{eV}$.

Assuming the weak coupling value of the superconducting gap $2 \Delta=3.5 k_{\mathrm{B}} T_{\mathrm{c}} \approx 7.5 \mathrm{meV}$ and using $v_{\mathrm{F}}=3 \times 10^{7} \mathrm{~cm} / \mathrm{s}$ leads to $\xi \approx 180 \AA$. Strong coupling corrections (likely, given the value of $T_{\mathrm{c}}$ ) would reduce this by less than a factor of two. From $\Lambda=0.13 \mu \mathrm{m}$, we obtain the ratio $\kappa=\Lambda / \xi=7$ indicating type-II superconductivity.

The Fermi surface (FS) consists of rounded triangular cylinders of mean radius $k_{\mathrm{F}}=\frac{2}{9} \frac{\pi}{a}$ centered at the corners $K$ of the hexagonal zone. Very low energy $\omega \rightarrow 0$ scattering processes are described by the phase space for scattering on the FS,

$$
\chi(\vec{Q})=\sum_{\vec{k}} \delta\left(\epsilon_{\vec{k}}\right) \delta\left(\epsilon_{\vec{k}+\vec{Q}}\right),
$$

where $\epsilon_{k}$ is the band dispersion relation and $E_{\mathrm{F}}$ is taken as the zero of energy. The processes consist of intra-FS scattering that would be discontinuous at $|\vec{Q}|=2 k_{\mathrm{F}}$ (causing the conventional Kohn anomalies) if the FS were circular, and inter-FS scattering between the cylinders centered at the inequivalent (but symmetry related) points $K$. If we denote the six 2D zone corners by $K_{1}, K_{2}, \ldots, K_{6}$ in counterclockwise order, these latter processes will be peaked only at wave vector $\vec{Q}=K_{1}-K_{2} \equiv K_{6}$ and symmetric wave vectors, since all other connecting wave vectors are reciprocal lattice vectors. For a circular FS $\chi(\vec{Q})$ diverges at $\vec{Q}=K$ due to exact nesting over circular FSs. The distortion of the FS into 3-fold symmetric cylinders will broaden the divergence into a peak. The scattering processes therefore are concentrated into a ring at $Q \approx 2 k_{\mathrm{F}}$ and peaks centered at the zone corners $K$. The widths of the peaks are related to the FS anisotropy, which is $\sim 20 \%$ of the mean Fermi wave vector.

We turn briefly to real space, where in fig. 4 we show a contour plot of the carrier (electron) density in a plane lying within one (dimpled) HfN layer. The magnitude is similar on Hf and $\mathrm{N}$, reflecting the strong hybridization. The maxima around the Hf ion avoid the nearby, highly 


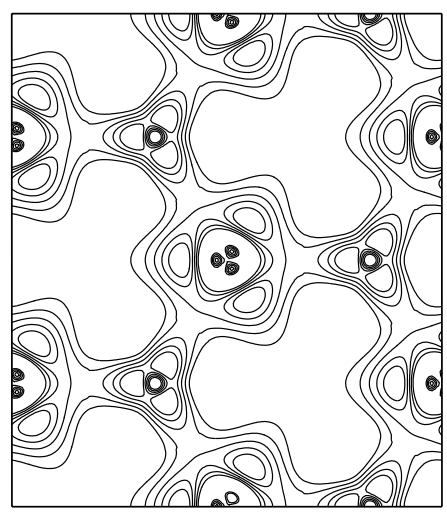

Fig. 4. - Contour plot illustrating the strong inhomogeneity of electron carrier density in $\mathrm{Na}_{0.25} \mathrm{HfNCl}$. Hf ions lie at the center and at the edges of the plot; $\mathrm{N}$ ions lie midway between three Hf ions. The plane of the figure lies between the $\mathrm{Hf}$ and $\mathrm{N}$ layers.

charged $\mathrm{N}^{3-}$ ions, and each triangle of Hf ions may be considered to form a weak three-center bond within the Hf plane. This density profile is very inhomogeneous and anisotropic within the plane, in spite of the large and nearly isotropic dispersion of the underlying states.

We have calculated the frequencies of the three fully symmetric $\left(A_{1 g}\right)$ Raman active vibrational modes of (undoped) $\mathrm{ZrNCl}$ and $\mathrm{HfNCl}$ at the zone center. These vibrations correspond to modulation of the internal coordinates $z_{\mathrm{Zr} / \mathrm{Hf}}, z_{\mathrm{N}}, z_{\mathrm{Cl}}$, and the structure must be relaxed before the phonon frequencies are calculated. We obtain $\omega=202,334,586 \mathrm{~cm}^{-1}$ for $\mathrm{ZrNCl}$ and 191, 386, $604 \mathrm{~cm}^{-1}$ for $\mathrm{HfNCl}$.

The highest frequency is dominated by $\mathrm{N}$ motion, and being in the $590-600 \mathrm{~cm}^{-1}$ range is similar to the highest oxygen mode in $\mathrm{La}_{0.85} \mathrm{Sr}_{0.15} \mathrm{CuO}_{4}$ [17]. The lower two modes are characterized by the $\mathrm{Zr}$ atom vibrating out of phase (respectively, in phase) with the $\mathrm{Cl}$ ion. The high- $N$ frequency is noteworthy, since coupling of phonons to high frequency modes is an important factor in enhancing $T_{\mathrm{c}}$ in conventional superconductors. However, in conventional superconductors a high value of $N\left(E_{\mathrm{F}}\right)$ is also required.

We have calculated the Hopfield parameter $\eta$ for $\mathrm{Na}_{0.25} \mathrm{HfNCl}$ using the usual GaspariGyorffy prescription [18]. The results are $\eta_{\mathrm{Hf}}=0.5 \mathrm{eV} / \AA^{2}, \eta_{\mathrm{N}}=0.2 \mathrm{eV} / \AA^{2}$, with $\mathrm{Cl}$ and $\mathrm{Na}$ contibutions entirely negligible. These values are one order of magnitude smaller than is typical in binary compounds with $T_{\mathrm{c}} \approx 20 \mathrm{~K}[19]$. The small values result from the combination of a small DOS and small phase shifts at the Fermi surface. Even allowing for an underestimate due to the non-close-packed structure and to high phonon frequencies (from $\mathrm{N}$ ), these values cannot account for the observed values of $T_{\mathrm{c}}$.

We summarize our main findings. Contrary to expectations this class of materials is a wide band, probably weakly correlated, electronic system. The wider gap (more insulating) parent compound corresponds to the higher- $T_{\mathrm{c}}$ superconductor, and the observed difference of $T_{\mathrm{c}}$ probably cannot be attributed solely to the higher vibrational frequencies. The light mass pairs form in a low density of states band of in-plane $d$ character that is symmetric across the HfN (ZrN) bilayer. The simple and distinctive Fermi surface concentrates scattering events that are involved in pairing into specific regions of wave vector. A combination of strong ionic and covalent bonding of the light $\mathrm{N}$ ion leads to high-energy phonon branches that might be expected to be important in the pairing, except that electron-phonon couplig seems to be weak. The origin of the high $T_{\mathrm{c}}$ remains mysterious. 
We are indebted to R. SEshadRI for many communications on the ZrNCl system, to comments from D. J. ScAlapino, and to S. Shamoto for communication of unpublished work including structural data. This research was supported by Office of Naval Research Grant No. N00014-97-1-0956 and National Science Foundation Grant DMR-9802076. RW acknowledges support from Fundación Antorchas Grant No A-13622/1-103.

\section{REFERENCES}

[1] Friend R. H. and Yoffe A. D., Adv. Phys., 36 (1987) 1.

[2] Kastner M. A., Birgeneau R. J., Shirane G. and Endoh Y., Rev. Mod. Phys., 70 (1998) 897.

[3] Erwin S. C. and Pickett W. E., Science, 254 (1991) 842; Erwin S. C., in Buckminsterfullerines, edited by W. E. Billups and M. A. Ciufolini (VCH Publ., Cambridge, UK) 1993, p. 217.

[4] Yamanaka S., Kawaji H., Hotehama K. and Ohashi M., Adv. Mater., 9 (1996) 771.

[5] Shamoto S., Kato T., Ono Y., Miyazaki Y., Ohoyama K., Ohashi M., Yamaguchi Y. and Kajitani T., Physica $C, 306$ (1998) 7.

[6] Yamanaka S., Hotehama K. and Kawaji H., Nature, 392 (1998) 580.

[7] Shamoto S., Iizawa K., Yamada M., Ohoyama K., Yamaguchi Y. and Kajitani T., J. Phys. Chem. Solids, 60 (1999) 1431.

[8] Ohashi M., Yamanaka S. and Hattori M., J. Ceram. Soc. Jpn. Int. Ed., 97 (1989) 1175.

[9] For $\mathrm{Na}_{0.29} \mathrm{HfNCl}$ the structure is rhombohedral $R \overline{3} m$ (\#166), with $a=3.5892 \AA$, $c=29.722 \AA$. Internal coordinates for the atoms (each in $6 c$ sites) are $z_{\mathrm{Hf}}=0.2083, z_{\mathrm{N}}=0.1368, z_{\mathrm{Cl}}=0.3944$. Na was refined in the $3 a$ site at the origin. $\mathrm{Li}_{0.16} \mathrm{ZrNCl}$ is isomorphous, with $a=3.60 \AA$ being slightly larger.

[10] Blaha P., Schwarz K. and Luitz J., WIEN97, Vienna University of Technology, 1997. Improved and updated version of the original copyrighted WIEN code, which was published by Blaha P., Schwarz K., Sorantin P. and Trickey S. B., Comput. Phys. Commun., 59 (1990) 399. The sphere radii used in fixing the LAPW basis were chosen to be 2.00 a.u for $\mathrm{Hf}(\mathrm{Zr}), \mathrm{Cl}$, and alkali atoms, and 1.90 for $\mathrm{N}$. Local orbitals (Zr $3 s, 3 p$; Hf $3 s, 3 p, 4 f ; \mathrm{N}$ and $\mathrm{Cl} 2 s$ ) were added to the basis set for extra flexibility and to allow semicore states to be treated within the same energy window as the band states. The LAPW plane wave cutoff corresponded to energy of 17.7 Ryd.

[11] Vanderbilt D., Phys. Rev. B, 32 (1985) 8412; Laasonen K., Pasquarello A., Car R., Lee C. and Vanderbilt D., Phys. Rev. B, 47 (1993) 10142.

[12] Filippetti A., Pickett W. E. and Klein B. M., Phys. Rev. B, 59 (1999) 7043.

[13] Filippetti A. and Pickett W. E., Phys. Rev. B, 59 (1999) 8397.

[14] Ceperley D. M. and Alder B. J., Phys. Rev. Lett., 45 (1980) 566, as parametrized by Perdew J. P. and Wang Y., Phys. Rev. B 45, (1992) 13244 or by Vosko S. H., Wilk L. and Nusair M., Can. J. Phys., 58 (1980) 1200.

[15] Perdew J. P., Burke S. and Ernzerhof M., Phys. Rev. Lett., 77 (1996) 3865.

[16] Felser C. and Seshadri R., J. Mater. Chem., 9 (1999) 459.

[17] Krakauer H., Pickett W. E. and Cohen R. E., Phys. Rev. B, 47 (1993) 1002.

[18] Gaspari G. and Gyorffy B. L., Phys, Rev. Lett., 29 (1972) 801.

[19] Klein B. M. and Pickett W. E., in Superconductivity in d-and f-Band Metals 1982, edited by W. BuCKEL and W. WEBER (Kernforschungszentrum Karlsruhe, Karlsruhe) 1982, p. 477. 